\title{
MicroRNA-21 stimulates epithelial-to-mesenchymal transition and tumorigenesis in clear cell renal cells
}

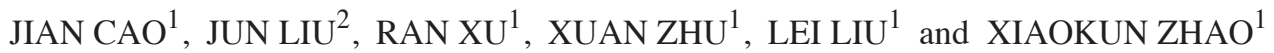 \\ ${ }^{1}$ Department of Urology, The Second Xiangya Hospital, Central South University, Changsha, Hunan 410011; \\ ${ }^{2}$ Department of Urology, The Fifth Teaching Hospital of Xinjiang Medical University, Wulumuqi, Xinjiang 830011, P.R. China
}

Received December 1, 2014; Accepted September 16, 2015

DOI: $10.3892 / \mathrm{mmr} .2015 .4568$

\begin{abstract}
Clear cell renal cell carcinoma (ccRCC) metastasis may result from epithelial-to-mesenchymal transition and mesenchymal stem cells that contribute to the development of the primary tumor. In this study, it was demonstrated that microRNA-21 (miR-21) acts as an oncogenic driver of ccRCC. ccRCC spheres were isolated and it was shown that they exhibited cancer stem cell-like properties, including the formation of self-renewing spheres. Spheres showed increased expression of stem cell-related transcription factors and epithelial-mesenchymal transition (EMT) markers. In addition, secondary sphere formation capacity was assessed after miR-21 transfection. miR-21 accelerated the formation of ccRCC spheres, which shared molecular characteristics with the spontaneous ccRCC spheres. It was demonstrated that miR-21 overexpression facilitates ccRCC sphere formation. Thus, a single miRNA may have an impact on the formation of highly tumorigenic cancer spheres in kidney cancer.
\end{abstract}

\section{Introduction}

Incidence of renal carcinoma is the third highest of all urinary system tumors, accounting for $\sim 3 \%$ of adult malignancies worldwide (1). Kidney cancer represents one of the 10 most common types of human cancer and is often resistant to chemotherapy. In addition, the morbidity and mortality of this type of cancer continues to increase (2). Approximately $70 \%$ of patients with renal cancer fall into the subgroup of clear cell renal cell carcinoma (ccRCC) (3) and a quarter of these patients have metastatic disease at initial diagnosis.

The short noncoding microRNAs (19-25 nucleotides) are an abundant class of small RNA that are important in gene regulation (4). MicroRNAs suppress protein expression by

Correspondence to: Professor Xiaokun Zhao, Department of Urology, The Second Xiangya Hospital, Central South University, 139 Middle Renmin Road, Changsha, Hunan 410011, P.R. China E-mail: zhaoxiaokun1955@sina.com

Key words: clear cell renal cell carcinoma, microRNA-21, cancer stem cell predominantly binding with imperfect complementarity to the 3' untranslated region of mRNAs to either repress translation or induce mRNA degradation (5). In a study using normal and ccRCC tumor tissues, miR-21 expression was increased in renal tumor tissue samples compared with adjacent nontumorous tissue samples (6). Although numerous mRNAs have been identified to be the targets of miR-21, few have been demonstrated to exhibit a specific role in tumorigenesis particularly in renal cancer.

The 'cancer stem cell' (CSC) model suggests that a small subset of cancer cells possess stem cell properties and are crucial in tumor initiation, tumor metastasis and resistance to anticancer therapy (7). CSC populations have been identified and characterized in a number of solid tumors, including melanoma, breast, brain, lung, colon, prostate, pancreatic and colorectal cancers (8). The characteristic features of CSCs include self-renewing properties, high tumorigenicity and the ability to differentiate into the cell types of the tumor of origin (9). Epithelial-mesenchymal transition (EMT) is important in inducing CSC formation in the epithelial cells of breast (10), colorectal (11), gastric (12) and prostate (13) cancers. Moreover, EMT was associated with increased expression of stem cell-related transcription factors and increased tumorigenic ability (14). The CSC model and EMT concepts have been integrated into a model which proposes that stationary CSCs are activated rendering them able to migrate to, and undergo EMT at the metastatic site (15).

In the present study, CSC-like cells with side population (SP) cell phenotype were isolated from human ccRCC cell lines to examine whether miRNAs contribute to the acquisition and maintenance of CSC characteristics in ccRCC. It was demonstrated that SP cells were highly clonogenic in vitro. These spheres showed increased expression of stem cell-related transcription factors and mesenchymal markers suggesting that EMT is a contributor to the gain of CSC features. Finally, it was identified that miR-21 overexpression results in the formation of cancer spheres.

\section{Materials and methods}

Patients and tissue samples. A total of $10 \mathrm{ccRCC}$ tissue samples and adjacent tissue samples were harvested from patients at the Second Xiangya Hospital (Changsha, China) between July 2013 and May 2014. The patients included 
5 females with an average age of 57 years old, and 5 males with an average age of 64 years old. The present study was approved by the ethics committee of the Second Xiangya Hospital, and written informed consent was obtained from the patients.

RT-qPCR analysis. TRIzol reagent (Invitrogen; Thermo Fisher Scientific, Inc.) was used to isolate total RNA from the snap frozen tissues. The isolated RNA was treated with DNase I (Invitrogen; Thermo Fisher Scientific, Inc.). The ratio of 28S/18S was analyzed by Glyko Bandscan 5.0 (Beijing Sage Creation Science Co., Ltd., Beijing, China). RNA quality and quantity were determined spectrophotometrically (Shanghai Spectrum Instruments Co., Ltd., Shanghai, China) at 260 and $280 \mathrm{~nm}$, respectively. RT-qPCR was performed as previously described $(16,17)$ using Roche Molecular Light Cycler (Roche Diagnostics, Mannheim, Germany). The specific reaction primers were as follows: $\beta$-actin forward, 5'-AGGGGCCGGACTCGTCATACT-3', and reverse, 5'-GGCGGCACCACCATGTACCCT-3'. The specific primers sets for miR-21 and U6 and the PCR mix were purchased from GeneCopoeia, Inc. (Rockville, MD, USA). The expression levels of U6 were used as an endogenous control. The reaction for each sample was performed in triplicate. Relative expression levels were calculated using the $2^{-\Delta \Delta \mathrm{Ct}}$ method (18).

Western blotting. To investigate EMT marker protein expression levels in cells, western blotting was performed. Protein concentrations were determined using a Bradford protein assay kit (Auragene Biosciences, Changsha, China). Protein $(100 \mu \mathrm{g})$ from each sample was loaded onto a $7.5 \%$ polyacrylamide gel (Amresco, LLC, Solon, OH, USA). Following electrophoresis, the SDS-PAGE separated proteins were transferred to a polyvinylidene difluoride membrane (Millipore, Billerica, MA, USA). The membrane was blocked with $5 \%$ non-fat milk in phosphate-buffered saline (PBS) and then incubated at room temperature for $3 \mathrm{~h}$ with the following primary antibodies: Monoclonal mouse anti-human fibronectin (cat. no. sc-271098; 1:500), polyclonal rabbit anti-human N-cadherin (cat. no. sc-7939; 1:200), polyclonal rabbit anti-human vimentin (cat. no. sc-5565; 1:500), polyclonal rabbit anti-human E-cadherin (cat. no. sc-7870; 1:1,000) purchased from Santa Cruz Biotechnology Inc. (Santa Cruz, CA, USA), or with monoclonal rabbit anti-human $\beta$-actin (cat. no. ab129348; 1:2,000; Abcam, Cambridge, MA, USA) in PBS for $3 \mathrm{~h}$. The membrane was then reprobed with polyclonal goat anti-mouse (ab6789; 1:5,000) and goat anti-rabbit (ab97051; 1:10,000) secondary antibodies (Abcam) conjugated with horseradish peroxidase for $40 \mathrm{~min}$ at room temperature. Blots were processed using an enhanced chemiluminescence kit (Santa Cruz Biotechnology Inc.) and exposed to film (Kodak, Rochester, NY, USA).

Cell culture and transfection assay. The 786-O and UOK-117 ccRCC cells were obtained from RIKEN BioResource Center (Ibaraki, Japan). Cells were cultured in RPMI-1640 medium (Gibco; Thermo Fisher Scientific, Inc.), supplemented with 10\% fetal bovine serum (FBS; Atlanta Biologicals, Lawrenceville, GA, USA), $100 \mathrm{U} / \mathrm{ml}$ penicillin and $100 \mu \mathrm{g} / \mathrm{ml}$ streptomycin at $37^{\circ} \mathrm{C}$ with $5 \% \mathrm{CO}_{2}$. $\mathrm{HK}-2$ cells were purchased from the
American Type Culture Collection (Manassas, VA, USA). Cells were cultured as a monolayer in Keratinocyte Serum Free Medium (K-SFM) supplemented with $0.05 \mathrm{mg} / \mathrm{ml}$ bovine pituitary extract, $5 \mathrm{ng} / \mathrm{ml}$ human recombinant epidermal grow th factor (EGF; Invitrogen; Thermo Fisher Scientific, Inc.) and $10 \%$ fetal bovine serum, $50 \mathrm{mg} / \mathrm{ml}$ penicillin and $50 \mathrm{mg} / \mathrm{ml}$ streptomycin (all from Invitrogen; Thermo Fisher Scientific, Inc.).

The miRNA inhibitors 2'-O-methyl antisense oligonucleotide targeting miR-21 (anti-miR-21), the miRNA inhibitor negative control (anti-miR-C), the pre-miR-21, and the mimic positive control were purchased from Shanghai GenePharma Co., Ltd. (Shanghai, China).

For transient transfection, a complex of Lipofectamine 2000 (Invitrogen Life Technologies) and plasmids or RNA oligonucleotides was prepared according to the manufacturer's instructions and directly combined with cells in 24-well cell culture plates at a density of $3 \times 10^{4}$ cells per well.

Determination of ccRCC cell proliferation. Cells were seeded at a density of 10,000 cells per well into 96-well culture plates, one day after transfection with lentivirus. Cells were further cultured for another 6, 24, 48 and $72 \mathrm{~h}$. To assess cell proliferation, cells were incubated with $20 \mu \mathrm{l}$ MTT at a final concentration of $0.5 \mathrm{mg} / \mathrm{ml}$ for $4 \mathrm{~h}$ at $37^{\circ} \mathrm{C}$. After removing the culture supernatant, $150 \mu 1$ dimethyl sulfoxide (DMSO, Beijing Solarbio Science \& Technology Co., Ltd. (Beijing, China) was added to solubilize the crystals for $20 \mathrm{~min}$ at room temperature. Absorbance was measured at a wavelength of $492 \mathrm{~nm}$ with a fluorescence plate reader (Molecular Devices, Sunnyvale, CA, USA).

Determination of ccRCC cell apoptosis. Apoptosis of ccRCC cells was induced by serum deprivation and cycloheximide (CHX; Sigma-Aldrich, St. Louis, MO, USA) treatment. UOK-117 and 786-O cells were pre-transfected with pre-miR-21 or anti-miR-21 and corresponding controls (50 nM), and then $24 \mathrm{~h}$ after transfection, cells were exposed to $\mathrm{CHX}$. Following a 4-h incubation with $\mathrm{CHX}$ at $37^{\circ} \mathrm{C}$, the cells were digested with trypsin (Beyotime Institute of Biotechnology, Shanghai, China) and collected prior to being washed twice with PBS. The cells $\left(1 \times 10^{6}\right)$ were then stained with Annexin V-fluorescein isothiocyanate and propidium iodide labeling solution (BD Biosciences, Franklin Lakes, NJ, USA), and then analyzed using a flow cytometer (Beckman Coulter, Brea, CA, USA) and an Annexin V/Dead Cell Apoptosis kit for flow cytometry (V13241, Invitrogen; Thermo Fisher Scientific, Inc.) according to the manufacturer's instructions.

Transwell assay. The migration and invasion of ccRCC cells were determined using a Transwell assay. Membranes $(8 \mathrm{~mm}$ filter) were embedded with collagen (Millipore). UOK-117 or $786-\mathrm{O}$ cells $\left(2.5 \times 10^{4}\right)$ were plated in Transwell chambers on the membrane. These chambers were placed in a $24-w e l l$ plate and incubated for $14 \mathrm{~h}$ at $37^{\circ} \mathrm{C}$. The cells that migrated/invaded through the membrane were stained with the reagent using a Crystal Violet Staining Solution kit (Beyotime Institute of Biotechnology). The stained cells were photographed using an inverted microscope (AE2000; Motic Incoporation, Ltd., Causeway Bay, Hong Kong). After capturing images, the 
A

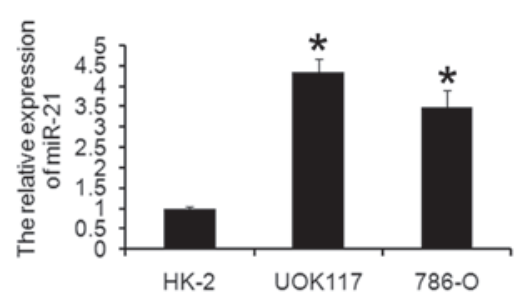

C

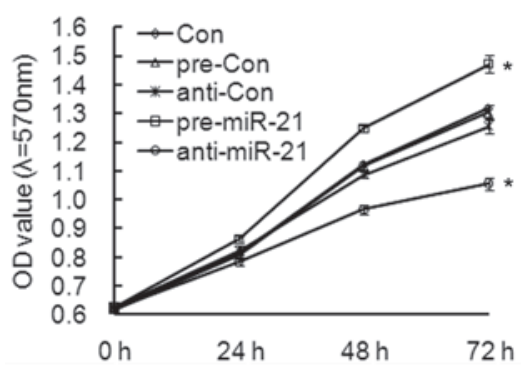

B 10.0 [DAdjacent tissues

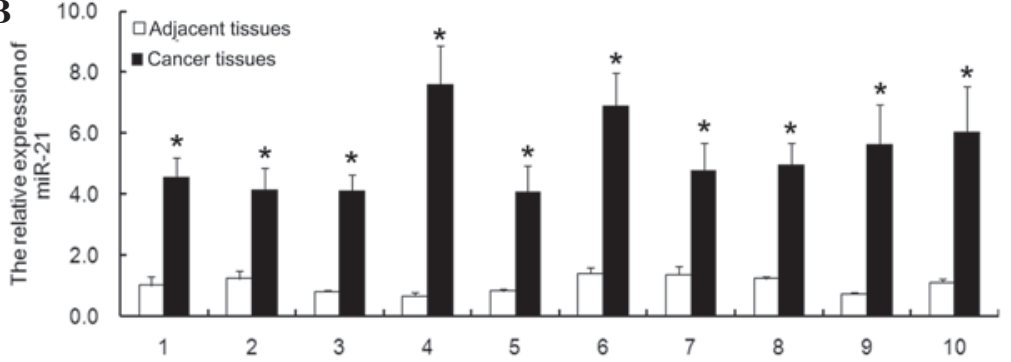

D

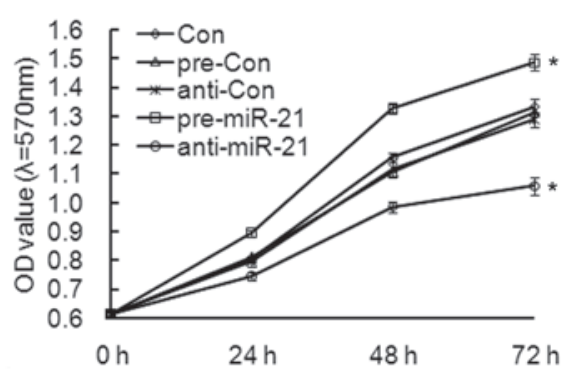

Figure 1. miR-21 expression is upregulated in ccRCC. (A) miR-21 expression was increased in UOK117 and 786-O ccRCC cell lines compared with the normal cells (HK-2). ${ }^{*} \mathrm{P}<0.05$, vs. HK-2. (B) miR-21 expression is increased in cancer tissues compared with adjacent tissues. ${ }^{*} \mathrm{P}<0.05$, vs. the adjacent tissue samples. An MTT assay revealed that miR-21 enhanced the proliferation ability of (C) UOK117 and (D) 786-O cells. Columns and bars, mean and standard deviation, respectively; ${ }^{*} \mathrm{P}<0.05$, vs. the control. ccRCC, clear cell renal cell carcinoma; miR, microRNA; OD, optical density.

stain from the membrane was eluted in DMSO according to the manufacturer's instructions. The absorbance of the eluted stain was measured with a spectrophotometer at $590 \mathrm{~nm}$. This measurement was used arbitrarily as an indicator of the number of cells that had migrated or invaded (19).

Wound scratch assay. Equal numbers of cells were plated in each well of twelve-well culture plates. After the cells reached $70-80 \%$ confluence, a line was scratched in the middle of the well using a pipette tip to create a wound. Images of the denuded area were captured using an inverted microscope (Motic Incoporation) just after the denudation (time, $0 \mathrm{~h}$ ). Following incubation for another $48 \mathrm{~h}$, images were again captured. For quantitation, the area of the wound was measured and normalized to vehicle-treated controls. To investigate the effects of miR-21 on migration of UOK-117 and 786-O cells, the cells were transfected with pre-miR-21 or anti-miR-21 and their respective negative controls for $24 \mathrm{~h}$, after which the wound was created and the images were captured at $0 \mathrm{~h}(20)$.

CSC sorting. CSC sorting and culture were performed as previously described (21-23). Briefly, under standard conditions, ccRCC cells were grown in RPMI-1640 (glucose $4.5 \mathrm{~g} / \mathrm{l}$ ) supplemented with L-glutamine, sodium pyruvate, $10 \%$ FBS and penicillin/streptomycin, in a humidified atmosphere at $37^{\circ} \mathrm{C}$ under $5 \% \mathrm{CO}_{2}$. Under sphere culture conditions, parental cell lines or the floating spheres obtained from transfections were plated in serum-free defined media (SFDM), which consisted of low-glucose (1 g/l) RPMI-1640 supplemented with L-Glutamine, sodium pyruvate, penicillin/streptomycin (Wisent Bio Products, Saint-Jean-Baptiste, QC, Canada), $20 \mathrm{ng} / \mathrm{ml}$ basic fibroblast growth factor (FGF), $20 \mathrm{ng} / \mathrm{ml}$ EGF, and B27 (Invitrogen; Thermo Fisher Scientific, Inc.) using 24-well ultra-low attachment plates (Corning Inc.,
Tewksbury, MA, USA). Spheres were dissociated with trypsin every 5-7 days and split to a 1:3 ratio. For the sphere formation assay, 500 cells/well were seeded in an ultra-low attachment 96-well plate (Corning Inc.) in $100 \mu 1$ SFDM/ well. SFDM ( $25 \mu 1 /$ well) was added every day for 3 weeks. Wells were photographed at $4 \mathrm{x}$ magnification (Nikon Eclipse TS100; Nikon, Tokyo, Japan). Sphere number was determined by ImageJ program, version 1.37 (National Institutes of Health, Bethesda, MA, USA).

Statistical analysis. Data are presented as the mean \pm standard deviation. Comparisons were made using one-way analysis of variance, and Student's t-test was used to analyze the differences between two experimental groups. Statistical analyses were performed using the SPSS version 17.0 software (SPSS, Inc., Chicago, IL, USA).. All experiments were repeated at least three times, and representative experiments are shown. $\mathrm{P}<0.05$ was considered to indicate a statistically significant difference.

\section{Results}

Expression of miR-21 is upregulated in ccRCC. The expression of miR-21 mRNA was examined in UOK-117 and 786-O ccRCC cells and normal HK-2 cells by RT-qPCR. miR-21 mRNA expression in ccRCC cells was significantly higher compared with that in the normal HK-2 cells (Fig. 1A).

Furthermore, the relative expression of miR-21 was compared between cancer tissues and adjacent tissues, and it was demonstrated that cancer tissues had higher miR-21 expression (Fig. 1B).

miR-21 promotes ccRCC cell proliferation and decreases apoptosis. To investigate the functional role of the upregulation 

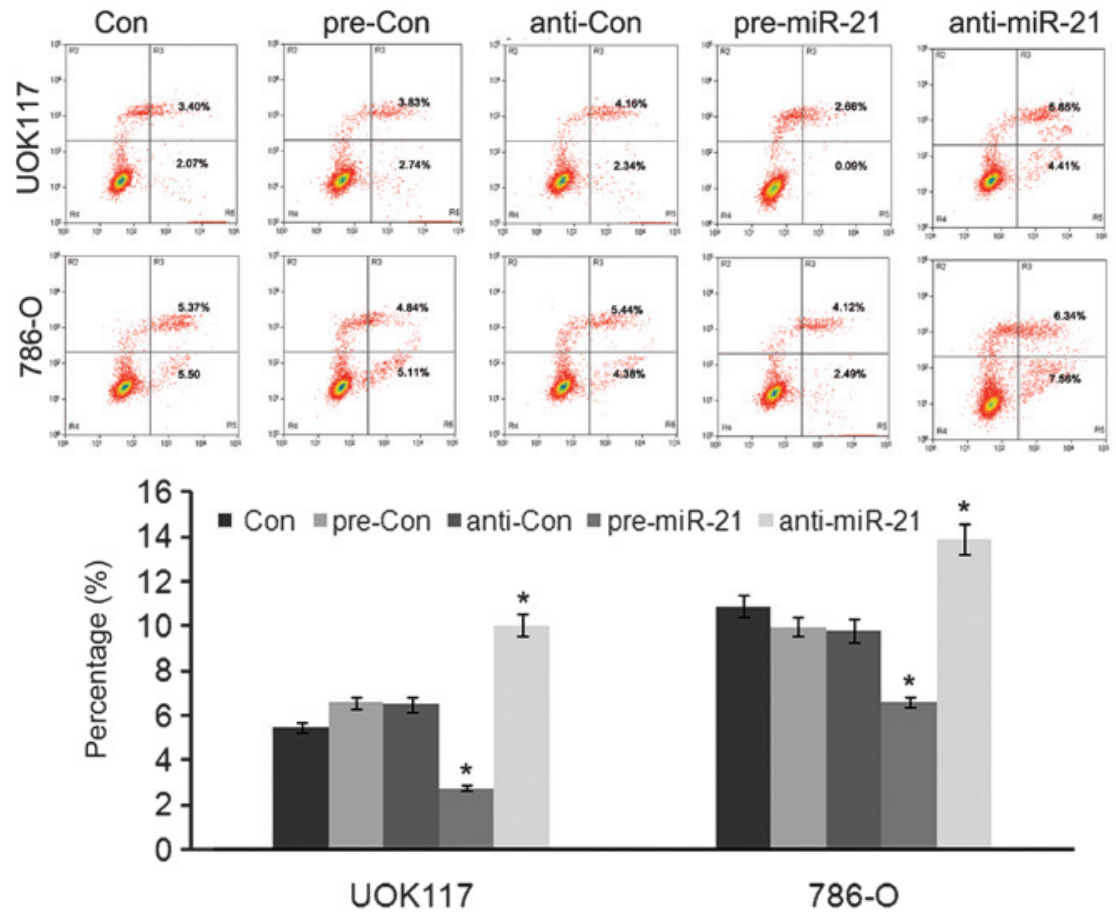

Figure 2. Flow cytometric analysis of apoptotic cells using Annexin V-fluorescein isothiocyanate. miR-21 inhibited the apoptosis of UOK117 and 786-O cells. Columns and bars, mean and standard deviation, respectively; " $\mathrm{P}<0.05$, vs. the control. ccRCC, clear cell renal cell carcinoma; miR, microRNA.
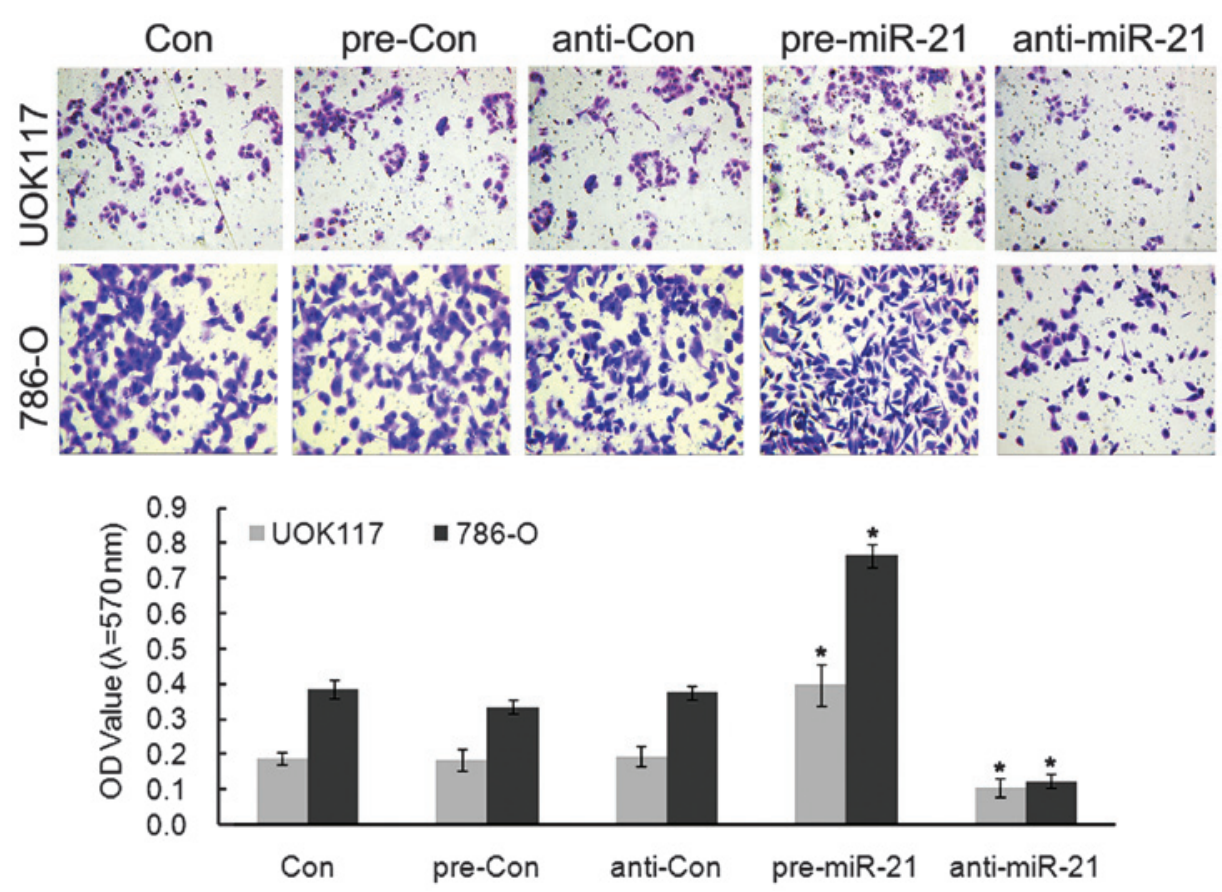

Figure 3. Transwell migration assays of ccRCC cells. miR-21 increased the cell invasion ability of UOK117 and 786-O cells. Columns and bars, mean and standard deviation, respectively; ${ }^{*} \mathrm{P}<0.05$, vs. the control. miR, microRNA; OD, optical density; ccRCC, clear cell renal cell carcinoma.

of miR-21 in ccRCC cells, pre-miR-21 or anti-miR-21 was transfected into ccRCC cells, and their biological behavior was examined. Cell proliferation was analyzed using the MTT assay and flow cytometric analysis. Transfection of pre-miR-21 induced significantly increased growth rates in UOK-117 and 786-O ccRCC cells (Fig. 1C and D), and it was clearly shown that the two types of ccRCC cells displayed a decreased apoptotic rate compared with the controls (Fig. 2) (24).
Effects of miR-21 overexpression on cell migration and invasion . Next, the role of miR-21 in regulating migration of ccRCC cells was determined using a Transwell chamber assay and wound scratch assay. Transfection with pre-miR-21 showed marked invasion, and anti-miR-21 blocked this invasion (Figs. 3 and 4). Quantification of these results showed significant changes in the ability to invade. Together these results demonstrated that miR-21 facilitates invasion of the two types of ccRCC cells (19). 


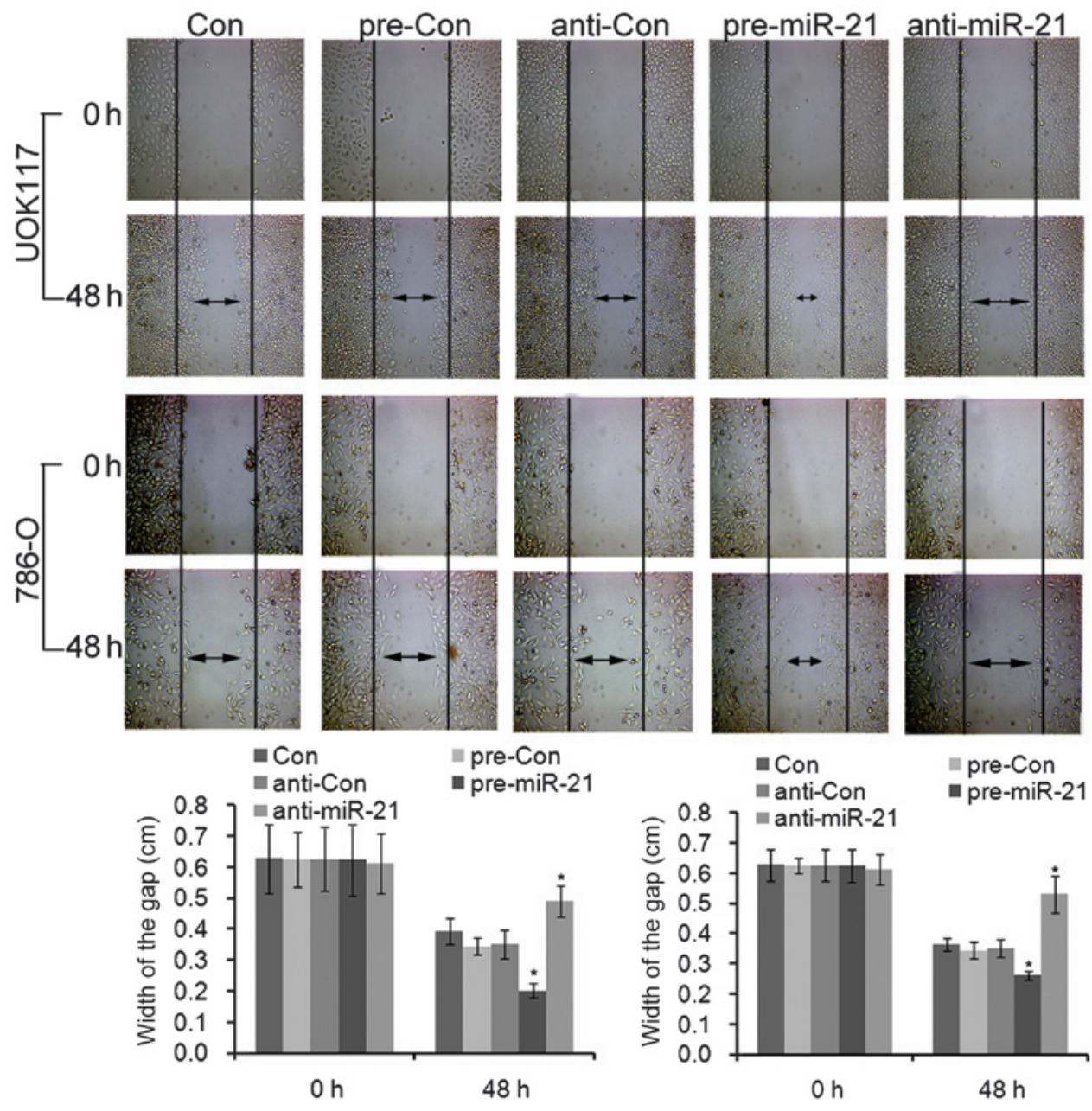

Figure 4. Wound healing assay of UOK117 and 786-O cells transfected with pre-miR-21 and anti-miR-21. miR-21 increased the cell migration ability of UOK117 and 786-O cells. Double arrow lines define the areas without cells. Columns and bars, mean and standard deviation, respectively; ${ }^{* * *} \mathrm{P}<0.05$, vs. the control.

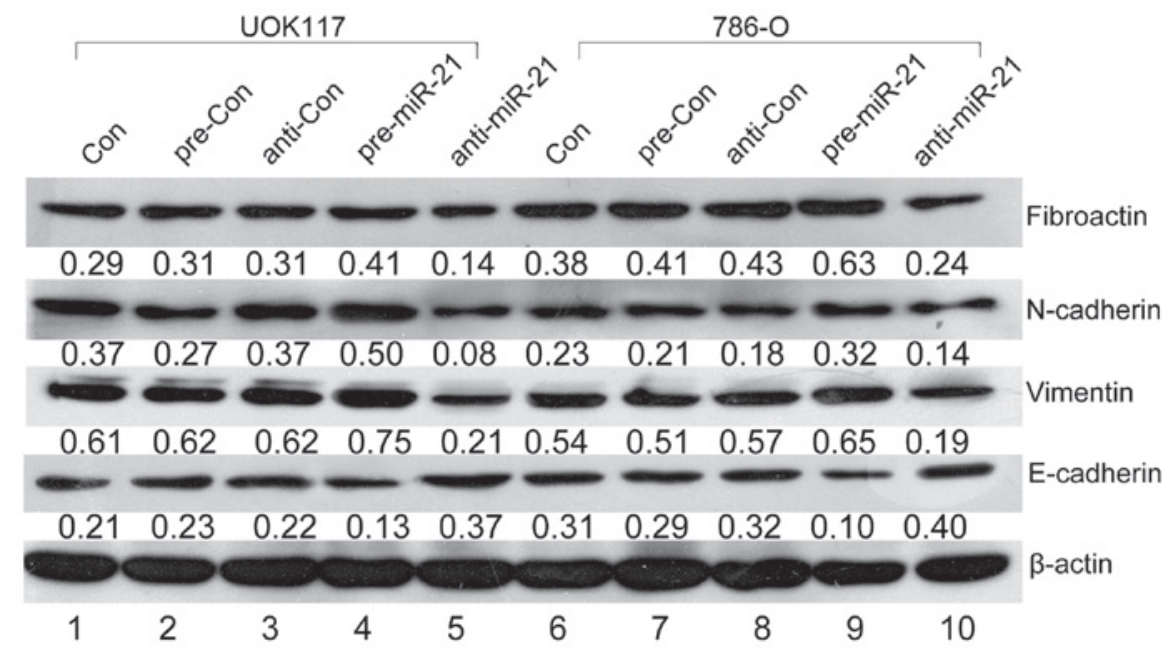

Figure 5. Western blot analysis of EMT markers, fibroactin, N-cadherin, vimentin and E-cadherin in UOK117 and 786-O cells. $\beta$-actin was used as control. EMT marker expression was increased after pre-miR-21 transfection in both cell types. EMT, epithelial-to-mesenchymal; miR, microRNA.

Cells overexpressing miR-21 exhibited accelerated invasion, as indicated in wound healing and invasion chamber assays. Overall, these data support miR-21 as an essential target for mediating the growth and invasiveness of ccRCC cells in vitro.
miR-21 enhances ccRCC cell EMT. UOK-117 and 786-O cells were transfected with pre-miR-21 or anti-miR-21. Western blotting was performed to determine EMT marker protein expression levels (fibroactin, $\mathrm{N}$-cadherin, vinmentin and 
A
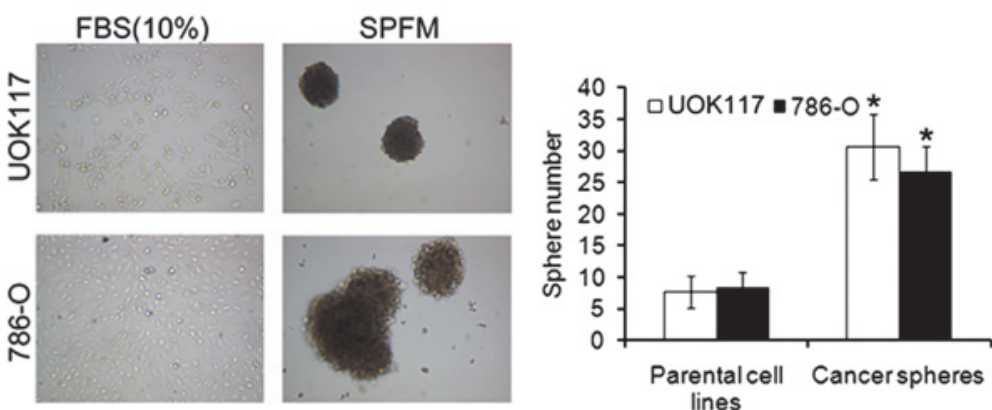

B
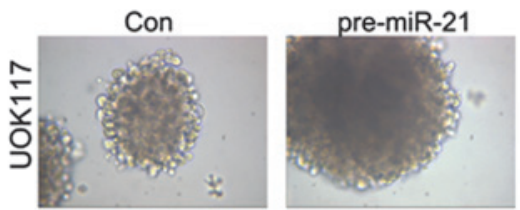

anti-miR-21
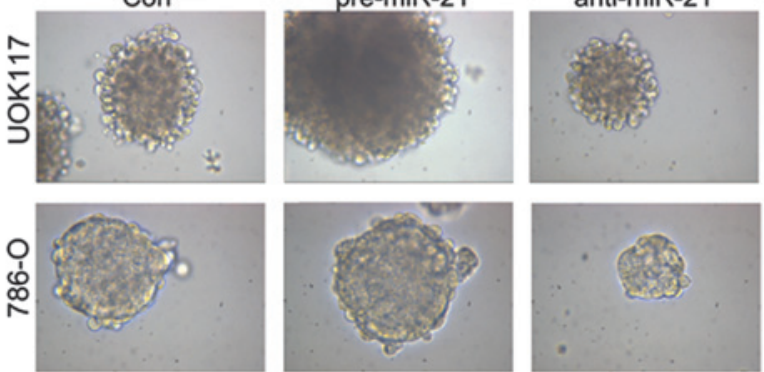

C

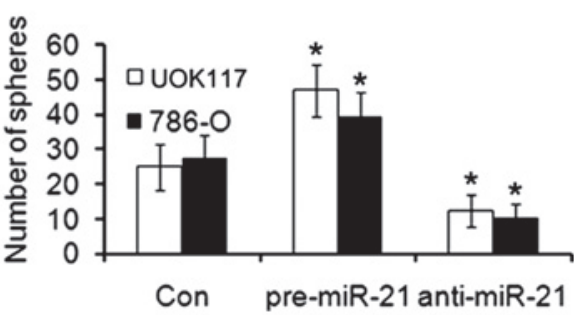

D

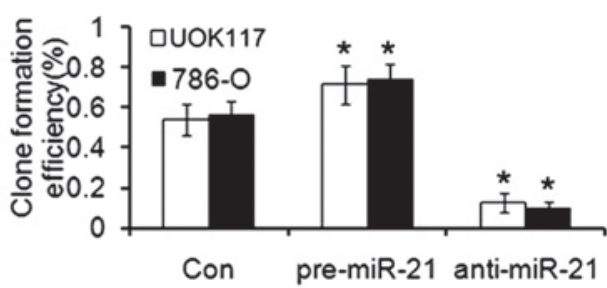

Figure 6. ccRCC cell lines form cancer spheres with stem cell-related features in SFDM and after pre-miR-21 transfection. (A) UOK117 and 786-O cells were propagated in SFDM, leading to the formation of 3D ccRCC spheres that could be propagated by enzymatic dissociation. Cells kept in regular medium (10\% FBS) did not form spheres. A sphere formation assay compared the sphere formation capacity of the parental cell lines and their 3D sphere derivatives. To assess the clonogenic potential of these sphere-forming cells, a single cell suspension prepared from ccRCC spheres and the parental cell lines was plated in SFDM. The sphere-derived cells typically formed $>3$-fold more spheres than the parental cell lines. (A) After single cell suspension, UOK117 and 786-O cell spheres were transfected with pre-miR-21 and anti-miR-21 (magnification, x100), the (B) sphere forming ability (magnification, $\mathrm{x} 400$ ), (C) sphere number, (D) clone formation efficiency of CSCs was observed. Columns and bars, mean and standard deviation, respectively. "P<0.05, vs. the control. 3D, three dimensional; ccRCC, clear cell renal cell carcinoma; SFDM, serum-free defined medium; miR, microRNA, FBS, fetal bovine serum.

E-cadherin). The levels of all the proteins were upregulated in UOK-117 and 786-O cells, compared with controls, respectively (Fig. 5). These data suggested that miR-21 can promote EMT of UOK-117 and 786-O cells.

Isolation of cancer spheres with stem cell properties from ccRCC cell lines. UOK-117 and 786-O cells were cultured in SFDM which was shown to support the formation of CSC spheres. Under these conditions, sphere formation was observed from the two ccRCC cell lines (Fig. 6A). Spheres could be propagated in three dimensional (3D) cultures in an ultra-low adherent dish for several passages. The ability of cell lines to form spheres in vitro depends on the presence of a self-renewing cell population. To assess the clonogenic potential of these sphere-forming cells, a single cell suspension prepared from UOK-117 and 786-O spheres and the parental cell lines was plated in SFDM. Large spheres were observed in 4-6 weeks. The sphere-derived cells typically formed $>2$ fold more spheres than the parental cell lines.

The role of miR-21 in cancer initiation and progression remains controversial. To determine the possible effect of miR-21 on ccRCC cancer sphere formation, ccRCC cell lines were transiently transfected with either pre-miR-21 or anti-miR-21. Transfection of UOK-117 and 786-O cells with pre-miR-21 led to rapid formation of 3D spheres (Fig. 6B), which were morphologically indistinguishable from the spheres obtained in SFDM (Fig. 6A). To confirm the ability of miR-21 to facilitate self-renewal, the clonogenic capacity of UOK-117 and 786-O cells was quantified upon miR-21 or treatment with transfection agent. miR-21 resulted in 1.9-fold increase in the number of colonies in the UOK-117 cell line and 1.5-fold increase in the number of colonies in the 786-O cell line compared with the transfection agent control (Fig. 6C and D).

\section{Discussion}

The current data investigated the expression of the recently identified tumor promoter miR-21, and demonstrated that miR-21 enhances the formation and/or stabilization of highly tumorigenic ccRCC spheres.

miR-21 was one of the first oncomiRs to be identified, and it has since been confirmed to be upregulated in numerous 
types of human cancer (25). In ccRCC, increased expression of miR-21 is associated with increased proliferation and invasion, and decreased apoptosis. Numerous studies have identified multiple miRNAs with potential prognostic or diagnostic use (26-36). Our data suggest that miR-21 was shown to be an independent prognostic factor for patients with ccRCC without metastasis at the time of diagnosis.

In recent years, research on CSCs in solid tumors have shown important findings. Baker et al (37) demonstrated that CSCs were a unique cell subpopulation, and a driving force for tumor growth in brain cancer, colon cancer and skin cancer. In this study, SP cells were isolated from human ccRCC cell lines to determine the biological properties of this cell population. A clone formation efficiency of SP cells higher than that of non-SP (NSP) cells was observed. These results provide direct evidence for the high tumorigenicity of SP cells. Self-renewal and multi-lineage differentiation capacities are hallmarks of stem cells. CSCs are considered to be able to undergo an asymmetrical self-renewing cell division, dividing into one stem cell and one progenitor cell, which could generate a variety of more differentiated functional cells that comprise the whole tumor society (38).

In conclusion, the data support the hypothesis that reduction of miR-21 expression may be able to to directly inhibit the proliferation of ccRCC cells. In addition, ccRCC spheres were isolated from two ccRCC cell lines and exhibited CSC-like properties including the formation of self-renewing spheres in serum-free defined media, high clonogenicity and increased expression of stem cell-specific transcription factors. In addition, it was also demonstrated that miR-21 may contribute to the acquisition of stem cell properties in metastatic ccRCC cell line models. The transfection of miR-21, enhanced the expression of EMT maker proteins, which was coupled with the presence of stem cell-like properties. The results of the present study suggested that miR-21 may be a promising new target for patients with ccRCC, which remains to be investigated in animal models.

\section{References}

1. Chow WH, Dong LM and Devesa SS: Epidemiology and risk factors for kidney cancer. Nat Rev Urol 7: 245-257, 2010.

2. Azeem K, Kollarova H, Horakova D, Magnuskova S and Janout V: Genetic syndromes associated with renal cell carcinoma: A review. Biomed Pap Med Fac Univ Palacky Olomouc Czech Repub 155: 231-238, 2011.

3. Novick AC: Kidney cancer: Past, present and future. Urol Oncol 25: 188-195, 2007.

4. Bartel DP: MicroRNAs: Genomics, biogenesis, mechanism and function. Cell 116: 281-297, 2004.

5. Bartel DP: MicroRNAs: Target recognition and regulatory functions. Cell 136: 215-233, 2009.

6. Yi Z, Fu Y, Zhao S, Zhang X and Ma C: Differential expression of miRNA patterns in renal cell carcinoma and nontumorous tissues. J Cancer Res Clin Oncol 136: 855-862, 2010.

7. Reya T, Morrison SJ, Clarke MF and Weissman IL: Stem cells, cancer and cancer stem cells. Nature 414: 105-111, 2001.

8. Visvader JE and Lindeman GJ: Cancer stem cells in solid tumours: Accumulating evidence and unresolved questions. Nat Rev Cancer 8: 755-768, 2008.

9. Prud'homme GJ: Cancer stem cells and novel targets for antitumor strategies. Curr Pharm Des 18: 2838-2849, 2012.

10. Han M, Liu M, Wang Y, Mo Z, Bi X, Liu Z, Fan Y, Chen X and $\mathrm{Wu} \mathrm{C}$ : Re-expression of miR-21 contributes to migration and invasion by inducing epithelial-mesenchymal transition consistent with cancer stem cell characteristics in MCF-7 cells. Mol Med Biochem 363: 427-436, 2012.
11. Fan F, Samuel S, Evans KW, Lu J, Xia L, Zhou Y, Sceusi E, Tozzi F, Ye XC, Mani SA and Ellis LM: Overexpression of snail induces epithelial-mesenchymal transition and a cancer stem cell-like phenotype in human colorectal cancer cells. Cancer Med 1: 5-16, 2012.

12. Kanzawa M, Semba S, Hara S, Itoh T and Yokozaki H: WNT5A is a key regulator of the epithelial-mesenchymal transition and cancer stem cell properties in human gastric carcinoma cells. Pathobiology : journal of immunopathology, molecular and cellular biology 80: 235-244, 2013.

13. Luo Y, Cui XH, Jiang YG, et al: [Epithelial-mesenchymal transition of prostate cancer: cancer stem cells or bulk cancer cells]. Zhonghua yi xue za zhi 93: 256-260, 2013.

14. Mani SA, Guo W, Liao MJ, Eaton EN, Ayyanan A, Zhou AY, Brooks M, Reinhard F, Zhang CC, Shipitsin M, et al: The epithelial-mesenchymal transition generates cells with properties of stem cells. Cell 133: 704-715, 2008.

15. Brabletz T, Jung A, Spaderna S, Hlubek F and Kirchner T: Opinion: Migrating cancer stem cells-an integrated concept of malignant tumour progression. Nat Rev Cancer 5: 744-749, 2005.

16. Nakamura T, Scorilas A, Stephan C, Yousef GM, Kristiansen G, Jung K and Diamandis EP: Quantitative analysis of macrophage inhibitory cytokine-1 (MIC-1) gene expression in human prostatic tissues. Br J Cancer 88: 1101-1104, 2003.

17. Cheung J, Mak YT, Papaioannou S, Evans BA, Fogelman I and Hampson G: Interleukin-6 (IL-6), IL-1, receptor activator of nuclear factor kappaB ligand (RANKL) and osteoprotegerin production by human osteoblastic cells: comparison of the effects of 17-beta oestradiol and raloxifene. J Endocrinol 177: 423-433, 2003

18. Livak KJ and Schmittgen TD: Analysis of relative gene expression data using real-time quantitative PCR and the 2(-Delta Delta C(T)) Method. Methods 25: 402-408, 2001.

19. Bera A, Das F, Ghosh-Choudhury N, Kasinath BS, Abboud HE and Choudhury GG: MicroRNA-21-induced dissociation of PDCD4 from rictor contributes to Akt-IKK $\beta$-mTORC1 axis to regulate renal cancer cell invasion. Exp Cell Res 328: 99-117, 2014.

20. Sheth S, Jajoo S, Kaur T, Mukherjea D, Sheehan K, Rybak LP and Ramkumar V: Resveratrol reduces prostate cancer growth and metastasis by inhibiting the Akt/MicroRNA-21 pathway. PLoS One 7: e51655, 2012.

21. Bornachea O, Santos M, Martinez-Cruz AB, García-Escudero R, Dueñas M, Costa C, Segrelles C, Lorz C, Buitrago A, Saiz-Ladera C, et al: EMT and induction of miR-21 mediate metastasis development in Trp53-deficient tumours. Sci Rep 2: 434, 2012.

22. Huang B, Huang YJ, Yao ZJ, Chen X, Guo SJ, Mao XP, Wang DH, Chen JX and Qiu SP: Cancer stem cell-like side population cells in clear cell renal cell carcinoma cell line 769P. PLoS One 8: e68293, 2013.

23. Lichner Z, Saleh C, Subramaniam V, Seivwright A, Prud'homme GJ and Yousef GM: MiR-17 inhibition enhances the formation of kidney cancer spheres with stem cell/tumor initiating cell properties. Oncotarget 6: 5567-5581, 2015.

24. Li L, Zhou L, Li Y, Lin S and Tomuleasa C: MicroRNA-21 stimulates gastric cancer growth and invasion by inhibiting the tumor suppressor effects of programmed cell death protein 4 and phosphatase and tensin homolog. J Buon 19: 228-236, 2014.

25. Selcuklu SD, Donoghue MT and Spillane C: MiR-21 as a key regulator of oncogenic processes. Biochem Soc Trans 37: 918-925, 2009.

26. Mitchell PS, Parkin RK, Kroh EM, Fritz BR, Wyman SK, Pogosova-Agadjanyan EL, Peterson A, Noteboom J, O'Briant KC, Allen A, et al: Circulating microRNAs as stable blood-based markers for cancer detection. Prco Natl Acad Sci USA 105: 10513-10518, 2008.

27. Brase JC, Johannes M, Schlomm T, Fälth M, Haese A, Steuber T, Beissbarth T, Kuner R and Sültmann H: Circulating miRNAs are correlated with tumor progression in prostate cancer. Int J Cancer 128: 608-616, 2011.

28. Zhang HL, Yang LF, Zhu Y, Yao XD, Zhang SL, Dai B, Zhu YP, Shen YJ, Shi GH and Ye DW: Serum miRNA-21: Elevated levels in patients with metastatic hormone-refractory prostate cancer and potential predictive factor for the efficacy of docetaxel-based chemotherapy. Prostate 71: 326-331, 2011.

29. Moltzahn F, Olshen AB, Baehner L, Peek A, Fong L, Stöppler H, Simko J, Hilton JF, Carroll P and Blelloch R: Microfluidic-based multiplex qRT-PCR identifies diagnostic and prognostic microRNA signatures in the sera of prostate cancer patients. Cancer Res 71: 550-560, 2011. 
30. Saini S, Majid S, Yamamura S, Tabatabai L, Suh SO, Shahryari V, Chen Y, Deng G, Tanaka Y and Dahiya R: Regulatory role of mir-203 in prostate cancer progression and metastasis. Clin Cancer Res 17: 5287-5298, 2011.

31. Collyer TC, Yates DR and Bellamy MC: Severe air embolism resulting from a perforated cap on a high-flow three-way stopcock connected to a central venous catheter. Eur J Anaesthesiol 24: 474-475, 2007.

32. Catto JW, Abbod MF, Wild PJ, Linkens DA, Pilarsky C, Rehman I, Rosario DJ, Denzinger S, Burger M, Stoehr R, et al: The application of artificial intelligence to microarray data: Identification of a novel gene signature to identify bladder cancer progression. Eur Urol 57: 398-406, 2010.

33. Neely LA, Rieger-Christ KM, Neto BS, Eroshkin A, Garver J, Patel S, Phung NA, McLaughlin S, Libertino JA, Whitney D and Summerhayes IC: A microRNA expression ratio defining the invasive phenotype in bladder tumors. Urol Oncol 28: 39-48, 2010.
34. Liu H, Brannon AR, Reddy AR, Alexe G, Seiler MW, Arreola A, Oza JH, Yao M, Juan D, Liou LS, et al: Identifying mRNA targets of microRNA dysregulated in cancer: With application to clear cell renal cell carcinoma. BMC Syst Biol 4: $51,2010$.

35. Liu W, Zabirnyk O, Wang H, Shiao YH, Nickerson ML, Khalil S, Anderson LM, Perantoni AO and Phang JM: miR-23b targets proline oxidase, a novel tumor suppressor protein in renal cancer. Oncogene 29: 4914-4924, 2010.

36. Catto JW, Alcaraz A, Bjartell AS, De Vere White R, Evans CP, Fussel S, Hamdy FC, Kallioniemi O, Mengual L, Schlomm T and Visakorpi T: MicroRNA in prostate, bladder and kidney cancer: A systematic review. Eur Urol 59: 671-681, 2011.

37. Baker M: Cancer stem cells tracked. Nature 488: 13-14, 2012.

38. Blagosklonny MV: Target for cancer therapy: Proliferating cells or stem cells. Leukemia 20: 385-391, 2006. 